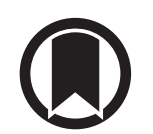

CrossMark

\title{
T-box protein 4 mutation causing pulmonary arterial hypertension and lung disease
}

\author{
To the Editor:
}

Pulmonary arterial hypertension (PAH) is a progressive disease mainly characterised by a widespread obstruction of distal pulmonary arteries [1]. Untreated, this disease rapidly leads to right heart failure, then to death [2]. Heritable PAH (hPAH) represents about 30\% of PAH cases, and this category includes familial forms with or without identified mutations, and sporadic forms carrying a mutation. The first genetic abnormalities discovered in hPAH were bone morphogenetic protein receptor-2 (BMPR2) mutations. Loss of function of this receptor results in proliferation of vascular smooth muscle cells and selection of endothelial cell clones resistant to apoptosis [3]. Since, several other mutations have been implicated in PAH. Mutation in the T-box protein 4 (TBX4) gene lead to an autosomal-dominant disorder called "small patella syndrome" or "coxopodopatellar syndrome", characterised by patellar aplasia and abnormalities of the feet and pelvis [4]. Recently, TBX4 mutation has been reported in childhood-onset $\mathrm{PAH}[5]$ and, more rarely, in adults [6, 7].

We report a case of a TBX4 mutation carrier presenting PAH but also bronchial and pulmonary parenchymal abnormalities potentially related to this mutation.

A 34-year-old woman presented in a tertiary care hospital with a potential diagnosis of severe PAH. She was a former smoker (5 pack-years) and had no medical history. The patient had progressive dyspnoea in New York Heart Association (NYHA) functional class III and she experienced right heart failure 2 months before admission.

Echocardiography revealed normal left ventricular function, a moderately dilated right ventricle and elevated systolic pulmonary artery pressure $(70 \mathrm{mmHg})$. Ventilation/perfusion lung scan ruled out venous thromboembolism disease. A pulmonary function test was unremarkable with the exception of a moderate to severe decrease in carbon monoxide diffusion capacity (53\% of the predicted value). Arterial blood gases showed a normal oxygen partial pressure $\left(\mathrm{PaO}_{2}\right)$ and hypocapnia. 6-min walk distance was $480 \mathrm{~m}$ with a decrease of oxygen saturation from $98 \%$ to $91 \%$. Right-sided heart catheterisation (RHC) diagnosed pre-capillary pulmonary hypertension (mean right atrial pressure $2 \mathrm{mmHg}$; pulmonary artery pressure (mean) 59/16 (35) $\mathrm{mmHg}$; pulmonary artery wedge pressure $6 \mathrm{mmHg}$; cardiac index $2.4 \mathrm{~L} \cdot \mathrm{min}^{-1} \cdot \mathrm{m}^{-2}$ and pulmonary vascular resistance (PVR) 7.2 Wood units). Chest computed tomography (CT) showed peripheral reticular lines, centrilobular nodules, thin-walled cysts that were prominent in the upper lobes and an appearance resembling diverticula in the trachea and large bronchi. Minimum intensity projection images highlighted a mosaic appearance showing distal airway involvement (figure 1).

Next generation sequencing-based targeted sequencing gene panel investigating predisposing genes for PAH found a copy number variant in TBX4 gene which is the complete deletion of the exon 5 (c.(549+1_550-1)_(702+1_703-1)del). Clinical examination showed shortened 4th and 5th feet arches without a "classic" small patella syndrome, and pelvis radiography revealed a lack of ossification in both ischia.

A diagnosis of heritable PAH was made, and she subsequently commenced treatment with a combination of an endothelin receptor antagonist and a phosphodiesterase-5 inhibitor.

@ERSpublications

A 34-year-old woman carrying a TBX4 mutation had pre-capillary pulmonary hypertension, emphysema like lesions and parenchymal lung fibrotic densification. TBX4 can be a complex cause of pulmonary hypertension in adults. http://bit.ly/2WLu0Rt

Cite this article as: Maurac A, Lardenois É, Eyries M, et al. T-box protein 4 mutation causing pulmonary arterial hypertension and lung disease. Eur Respir J 2019; 54: 1900388 [https://doi.org/10.1183/ 13993003.00388-2019]. 

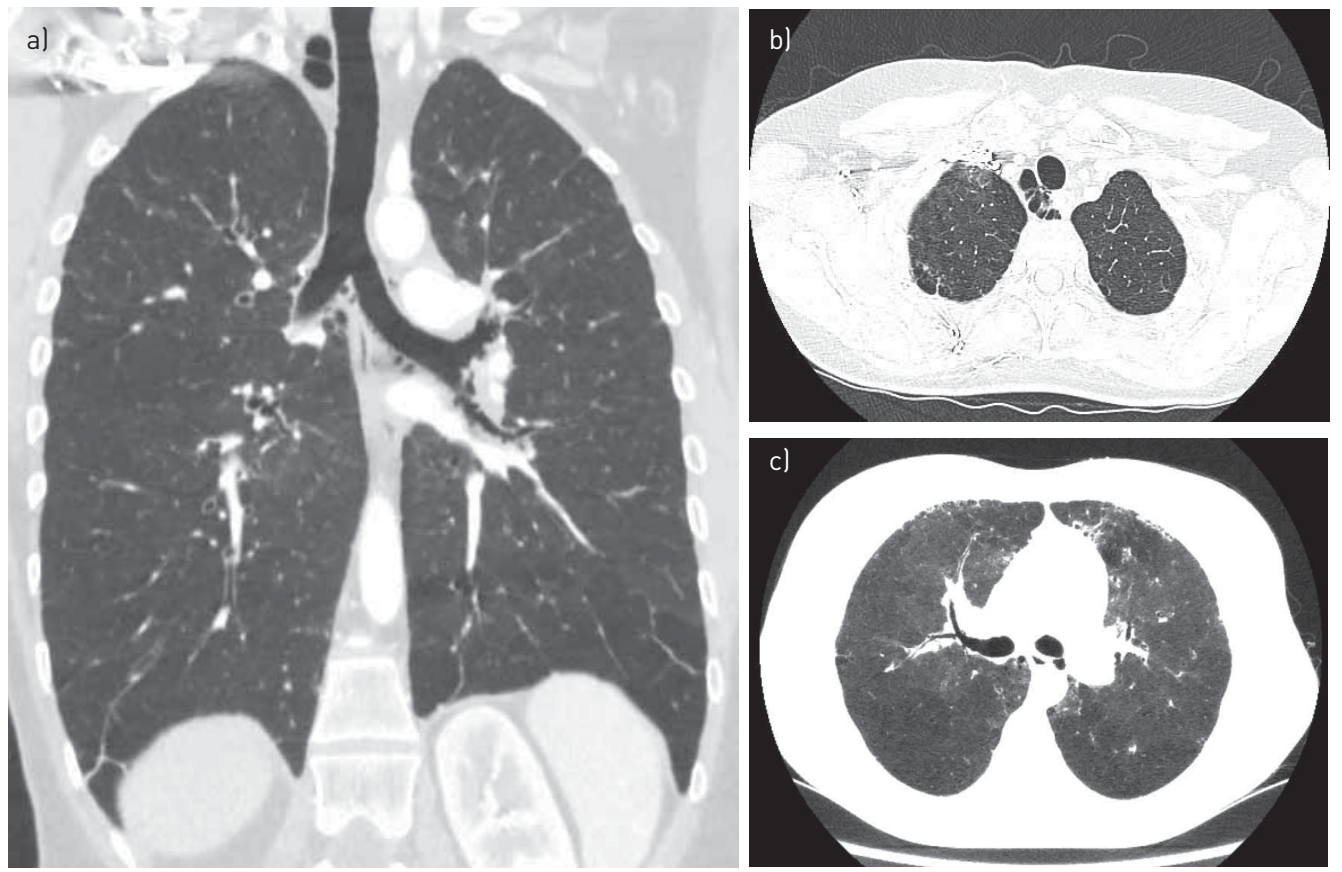

FIGURE 1 Chest computed tomography. a) Coronal plane at the level of the carina in lung window showing mainly many diverticula next to the trachea and large bronchi and a mosaic pattern of lung attenuation. b) Axial plane at the upper third of the lung in lung window showing parenchymal destruction resembling emphysema, few diverticula next to the trachea and few nodules in the right upper lobe. c) Axial minimum intensity projection reconstruction at the lower third of the lung showing parenchymal heterogeneity with mosaic attenuation pattern emphasising distal airway involvement.

Due to the development of a ptosis, a change of the voice and a pseudo-nodular thymus with hypermetabolic signs on positron-emission tomography, myasthenia gravis was suspected and a thymectomy was performed. During this surgery a lung biopsy from left upper lobe was performed.

Pathology of the lung showed fibrotic densification incorporating many cholesterol crystals located in the perivascular connective tissue (figure 2). Cholesterol clefts were found both within interstitium and in alveoli and were surrounded by numerous lymphocytes and foamy macrophages, without the formation of granuloma. No fibroblastic foci were noticed in lung biopsy. Dilated bronchi containing cell debris, macrophages and cholesterol clefts were enclosed by dense collagen bundles. Major vascular remodelling was observed within interstitial fibrosis. Pulmonary arteries were thickened, showing increase of the muscular media and intima. No blood clots were found in pulmonary vessels. No vascular inflammation was found, nor plexogenic arteriopathy.

Outside these foci, the parenchyma appeared emphysematous with thickened bronchiolar wall, characterised by expansion of muscular layer and mild fibrosis.

The patient responded partially to specific PAH drug therapy. At 4 months of treatment, NYHA functional class was unchanged (III), as well as 6-min walk distance $(525 \mathrm{~m})$. Brain-type natriuretic peptide was at $12 \mathrm{pg} \cdot \mathrm{mL}^{-1}$ and PVR decreased by $50 \%$ compared to baseline. After thymectomy, pulmonary haemodynamic measurements worsened then remained stable. The last RHC while the patient was receiving endothelin receptor antagonist and phosphodiesterase-5 inhibitor was performed 18 months after the diagnosis of PAH and showed a mean pulmonary artery pressure of $39 \mathrm{mmHg}$, cardiac index of 3.8 L $\cdot \mathrm{min}^{-1} \cdot \mathrm{m}^{-2}$ and PVR of 6.7 Wood units. Compared to the previous evaluation shown above, NYHA functional class and 6-min walk distance were unchanged.

The patient reported here had parenchymal pulmonary abnormalities, airway diverticula, small patella syndrome and PAH. The known role of TBX4 in lung development suggests that bronchial, lung parenchymal and pulmonary vascular abnormalities observed in this patient are at least in part related to TBX4 mutation.

Although chest CT and lung pathology showed pulmonary parenchymal abnormalities, we eliminated group 3 pulmonary hypertension. According to current knowledge, several criteria were in favour of group 1 pulmonary hypertension (i.e. a $\mathrm{PAH}$ ): forced expiratory volume in $1 \mathrm{~s}$, forced vital capacity and $\mathrm{PaO}_{2}$ 

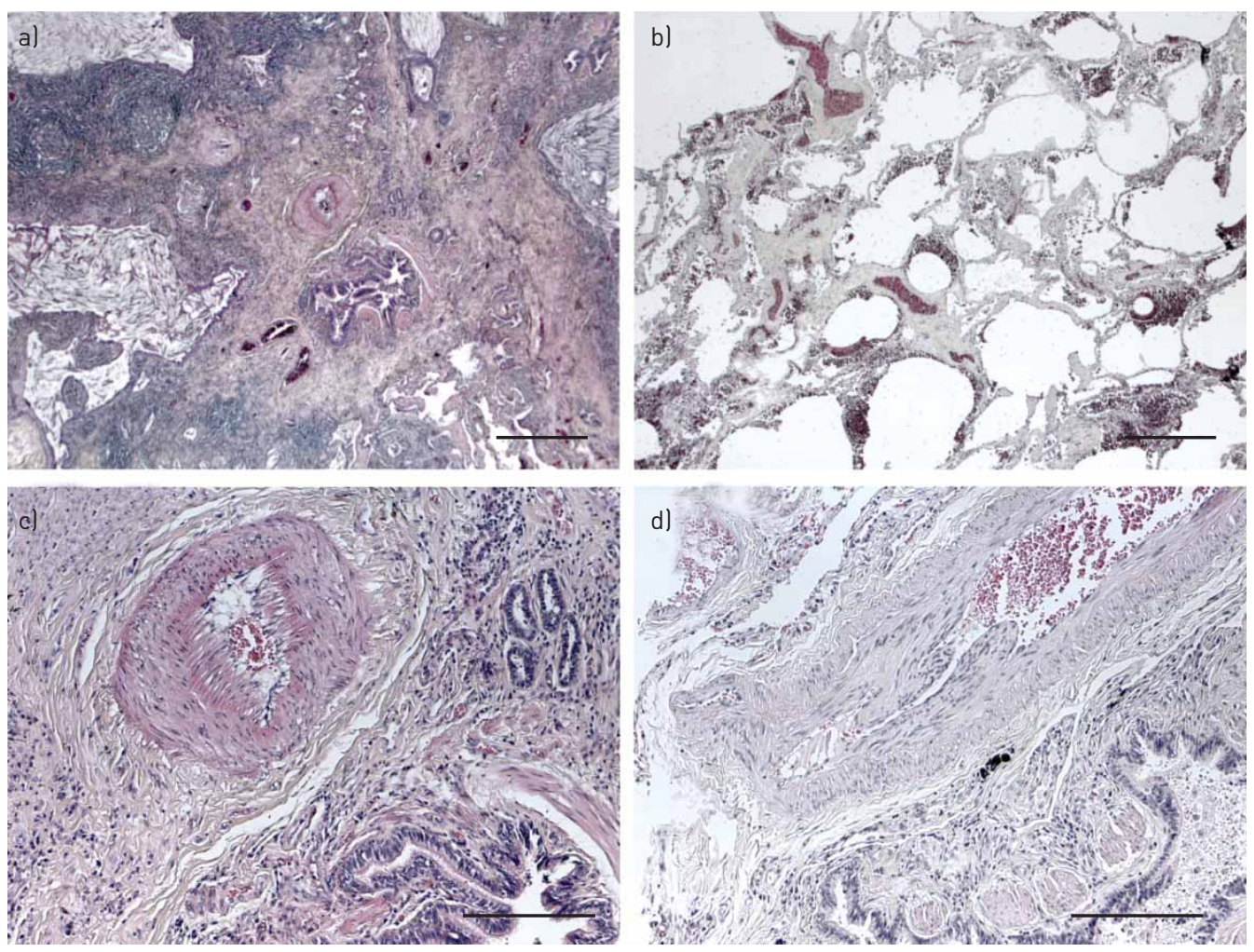

FIGURE 2 Pathology of biopsy specimen of the upper lobe of the left lung. a) Fibrotic densification of the lung parenchyma, with accumulation of endogenous cholesterol clefts and inflammatory reaction. A marked remodelled pulmonary artery is noticed within fibrosis. b) Altered lung parenchyma: emphysema-like lesions next to fibro-inflammatory foci. Scale bars $500 \mu \mathrm{m}$. $c$ and d) Pulmonary artery remodelling is characterised by the increase of both muscular media and intima. There is neither plexiform lesion nor thrombosis. Scale bars $200 \mu \mathrm{m}$.

were within the normal range [8]. However, in the light of recent publications showing severe lung development abnormalities in infants, as well as the present case observed in an adult, the position of pulmonary hypertension in patients with a TBX4 mutation in the clinical classification is likely to be reassessed $[9,10]$.

TBX4 belongs to the T-box family of transcription factors and is characterised by a DNA-binding motif known as the T-domain. TBX4 is expressed in many tissues, including lung and bone [11]. In addition to the bone involvement, subjects who are heterozygous for TBX4 may develop PAH $[5,6]$. The penetrance of PAH in TBX4 mutation carriers is largely unknown and such a mutation is an uncommon genetic cause of heritable PAH in adults [7]. The long-term response to the specific treatment of PAH in these patients is also unknown.

Diverticula on the trachea and main bronchi visible on chest CT and densification foci of the lung parenchyma with cholesterol crystals on lung biopsy in the patient presented here may be consistent with a lack of airway continuity [12]. Two recent publications describing three newborns with a TBX4 mutation, two having a coxopodopatellar syndrome and who developed respiratory failure at birth suggests that our patient's lung abnormalities may be related at least partially to a lung development disorder $[9,13]$. Alternatively, patients with TBX4 mutations may be more susceptible to environmental exposure, such as cigarette smoking. Thus, the association of severe pre-capillary pulmonary hypertension and unusual lung parenchymal disease should lead to suspicion of TBX4 mutation.

It has been shown in vitro that lung development is dependent on transcription factors TBX4 and TBX5 [14]. Complex compound inheritance including TBX4 mutation was found in 10 out of 26 individuals having rare lethal lung hypoplasia [10]. Although chest CT and results of the lung biopsy in our patient do not demonstrate an alteration of lung development, we suspect such a process from the known role of TBX4.

We report a case of heritable PAH due to TBX4 mutation with parenchymal lung abnormalities possibly due to an alteration of the process of lung development. Given that dyspnoea on exertion is a common sign of both PAH and parenchymal lung or bronchial diseases, we suggest a careful lung assessment on 
chest CT in heritable PAH due to TBX4 mutation. Conversely, it also seems important from a clinical point of view not to ignore pulmonary vascular disease when pulmonary parenchymal involvement is obvious.

Arnaud Maurac ${ }^{1}$, Émilie Lardenois ${ }^{2}$, Mélanie Eyries ${ }^{3}$, Maria Rosa Ghigna ${ }^{4}$, Isabelle Petit ${ }^{5}$, David Montani $\oplus^{6}$, Anne Guillaumot $^{1}$, Brice Caput ${ }^{7}$, François Chabot ${ }^{1,8}$ and Ari Chaouat $\circledast^{1,8}$

${ }^{1}$ Pôle des spécialités médicales/département de pneumologie, CHRU de Nancy, Vandouvre-lès-Nancy, France. ${ }^{2}$ Laboratoire d'anatomie et cytologie pathologiques, CHRU de Nancy, Vandœuvre-lès-Nancy, France. ${ }^{3}$ Département de génétique, hôpital Pitié-Salpêtrière, Assistance Publique-Hôpitaux de Paris (AP-HP), Paris, France. ${ }^{4}$ Pathology Dept, Marie Lannelongue Hospital, INSERM UMR_S 999, Le Plessis-Robinson, France. ${ }^{5}$ Département de radiologie, CHRU de Nancy, Vandœuvre-lès-Nancy, France. ${ }^{6}$ Univ. Paris-Sud, AP-HP, Centre de Référence de l'Hypertension Pulmonaire, Service de Pneumologie, INSERM UMR_S999, Hôpital de Bicêtre, Le Kremlin Bicêtre, France. ${ }^{7}$ Service de chirurgie thoracique, CHRU de Nancy, Vandœuvre-lès-Nancy, France. ${ }^{8}$ Université de lorraine, Inserm, U1116, Vandœuvre-lèsNancy, France.

Correspondence: Ari Chaouat, Pôle des spécialités médicales/département de pneumologie, CHRU de Nancy, rue du Morvan, 54500 Vandœuvre-lès-Nancy, France. E-mail: a.chaouat@chru-nancy.fr

Received: March 112019 | Accepted after revision: May 052019

Conflict of interest: A. Maurac has nothing to disclose. É. Lardenois has nothing to disclose. M. Eyries has nothing to disclose. M.R. Ghigna has nothing to disclose. I. Petit has nothing to disclose. D. Montani reports grants and personal fees from Actelion and Bayer, personal fees from GSK, MSD and Pfizer, outside the submitted work. A. Guillaumot has nothing to disclose. B. Caput has nothing to disclose. F. Chabot has nothing to disclose. A. Chaouat has nothing to disclose.

\section{References}

1 Dorfmüller P. Pathology of pulmonary vascular diseases. In: Pulmonary Circulation. 3rd Edn. Peacock AJ, Naeije R, Rubin LJ, eds. Boca Raton, CRC Press, 2011; pp. 29-45.

2 Galiè N, Humbert M, Vachiery J-L, et al. 2015 ESC/ERS Guidelines for the diagnosis and treatment of pulmonary hypertension: The Joint Task Force for the Diagnosis and Treatment of Pulmonary Hypertension of the European Society of Cardiology (ESC) and the European Respiratory Society (ERS). Eur Respir J 2015; 46: 903-975.

3 Soubrier F, Chung WK, Machado R, et al. Genetics and genomics of pulmonary arterial hypertension. J Am Coll Cardiol 2013; 62: D13-D21.

4 Bongers EMHF, Duijf PHG, van Beersum SEM, et al. Mutations in the human TBX4 gene cause small patella syndrome. Am J Hum Genet 2004; 74: 1239-1248.

5 Levy M, Eyries M, Szezepanski I, et al. Genetic analyses in a cohort of children with pulmonary hypertension. Eur Respir J 2016; 48: 1118-1126.

6 Gräf $\mathrm{S}$, Haimel $\mathrm{M}$, Bleda $\mathrm{M}$, et al. Identification of rare sequence variation underlying heritable pulmonary arterial hypertension. Nat Commun 2018; 9: 1416.

7 Eyries M, Montani D, Nadaud S, et al. Widening the landscape of heritable pulmonary hypertension mutations in pediatric and adult cases. Eur Respir J 2019; 53: 1801371.

8 Nathan SD, Barbera JA, Gaine SP, et al. Pulmonary hypertension in chronic lung disease and hypoxia. Eur Respir J 2019; 53: 1801914.

9 Suhrie K, Pajor NM, Ahlfeld SK, et al. Neonatal lung disease associated with TBX4 mutations. J Pediatr 2019; 206: 286-292.e1.

10 Karolak JA, Vincent M, Deutsch G, et al. Complex compound inheritance of lethal lung developmental disorders due to disruption of the TBX-FGF pathway. Am J Hum Genet 2019; 104: 213-228.

11 Papaioannou VE. The T-box gene family: emerging roles in development, stem cells and cancer. Development 2014; 141: 3819-3833.

12 Betancourt SL, Martinez-Jimenez S, Rossi SE, et al. Lipoid pneumonia: spectrum of clinical and radiologic manifestations. AJR Am J Roentgenol 2010; 194: 103-109.

13 Szafranski P, Coban-Akdemir ZH, Rupps R, et al. Phenotypic expansion of TBX4 mutations to include acinar dysplasia of the lungs. Am J Med Genet A 2016; 170: 2440-2444.

14 Cebra-Thomas JA, Bromer J, Gardner R, et al. T-box gene products are required for mesenchymal induction of epithelial branching in the embryonic mouse lung. Dev Dyn 2003; 226: 82-90. 